\title{
ARTE Y ARQUITECTURA: UNA PERSPECTIVA GNOSEOLÓGICA PARA NUESTRO TIEMPO
}

\section{Iskandar Rementeria Arnaiz}

Universidad del País Vasco / Euskal Herriko Unibertsitatea. Dpto. de Didáctica de la Expresión Musical, Plástica y Corporal. Grupo de Investigación GIC IT1096/16-21

\section{Resumen}

La arquitectura supone el terreno desde el cual poder reflexionar y reconocer las complejidades que el arte encierra en su relación con el espacio público y la comunidad. Sin embargo, no es baladí que ciertas líneas del pensamiento moderno y tardomoderno, mediante sus categorizaciones, pusieran atención a las relaciones entre arte y arquitectura desde una perspectiva que convendremos en denominar como gnoseológica, que reconocería tanto las particularidades disciplinares como la condición específica del saber creador y su posición en relación con los demás saberes conformadores de una sociedad. Tras el fracaso político del proyecto moderno, y en un escenario cada vez más complejo en el que la cultura y el espacio público suponen significantes empleados en las estrategias de inserción en las nuevas economías, estas relaciones podrían suponer, de nuevo, tanto las bases de las redefiniciones a las que el arte y la arquitectura se ven sometidas debido a los cambios epocales, como al reconocimiento del espacio común al que aspiran.

\section{Palabras clave: ARTE, ARQUITECTURA, GNOSEOLOGÍA, ESPACIO COMÚN, OTEIZA}

\section{ART AND ARCHITECTURE: A GNOSEOLOGICAL PERSPECTIVE FOR OUR TIME}

\begin{abstract}
Architecture is the terrain from which to reflect and recognize the complexities that art contains in its relationship with public space and the community. However, it is not trivial that certain lines of modern and late-modern thought, through their categorizations, paid attention to the relationships between art and architecture from a perspective that we shall agree to call gnoseological, which would recognize both the disciplinary particularities and the specific condition of creator knowledge and its position in relation to the other knowledge that composes a society. After the political failure of the modern project, and in an increasingly complex scenario in which culture and public space represent significant used for the new economies, these relationships could once again represent both the bases of the redefinitions to which art and architecture are subjected due to epochal changes, as well as the recognition of the common space to which they aspire.
\end{abstract} Keywords: ART, ARCHITECTURE, GNOSEOLOGY, COMMON SPACE,
OTEIZA

\footnotetext{
Rementeria Arnaiz, Iskandar. 2020. "Arte y arquitectura: Una perspec. tiva gnoseológica para nuestro tiempo”. AusArt 8 (2): 15-24. D0I: 10.1387 /ausart.22104
}

\section{AUSART}




\section{ARTE Y ARQUITECTURA EN LAS NUEVAS ECONOMÍAS}

Las últimas dos décadas nos han procurado la posibilidad de observar los modos en los que arte y arquitectura toman lugar en programas de revitalización y regeneración de espacios urbanos: museos al aire libre para el paseo y zonas de esparcimiento en la ciudad; centros de arte contemporáneo en zonas degradadas como elementos para una "revitalización", gentrificación y transformación urbana en los denominados "barrios creativos"; incluso procesos participativos para la transformación urbanística de las ciudades en los que se observa cierta estetización de la sociología para una supuesto agenciamiento de los ciudadanos. Sin embargo, los éxitos o fracasos de estas acciones no vienen determinados por los objetivos planteados en los discursos de las instancias que los animan, sino más bien en el cumplimiento de indicadores específicos, más sencillos de analizar a corto plazo que el efecto que en sus propios términos el arte y la arquitectura pudieran tener de forma efectiva en la comunidad.

En el ámbito concreto de Bilbao, los discursos vinculados a lo que las instituciones denominaron la "segunda revolución"1 de esta ciudad, colocaba a la arquitectura y al arte como elementos cruciales en la transformación de la ciudad, aunque en aquel momento se estuviera produciendo sin atender en demasía a la realidad artística del País Vasco, y tuviera como objetivo real la creación de una imagen de ciudad atractiva para el turismo; una transformación que, sin embargo, habría sido más efectiva no tanto por la influencia directa del arte y la arquitectura como a las operaciones de cambios de uso del suelo.

Aunque el caso de Bilbao tuviera sus particularidades y supusiera un modelo para otras ciudades, dichas estrategias institucionales correspondían a una tendencia global caracterizada por la fusión de las esferas cultural y económica propia de la lógica posmoderna, que en la ciudad vasca se adoptó tras la necesidad de cambio de modelo productivo derivada de los procesos de Transición política en el Estado español durante la década de 1980.

La apertura del museo Guggenheim en 1997 supuso la total inserción en tales lógicas, que entienden la ciudad misma -y su imagen- como un "sector de futuro", si bien los años previos habrían supuesto un verdadero gozne en lo que al cambio de paradigma se refiere. No obstante, como ya sabemos, la posmodernidad difícilmente consiguió o deseó desembarazarse de algunos 
de los presupuestos modernos, y las relaciones o remisiones a la modernidad desde el nuevo paradigma encontraron distintas vías. En el caso de instancias públicas y privadas adalides del nuevo cambio, pudo observarse la apropiación del discurso emancipatorio surgido del arte y la arquitectura durante el proyecto moderno para su uso en la legitimación de estrategias urbanas con el fin de incorporarse en las nuevas economías.

La siguiente frase resulta ilustrativa: "La transformación es un producto de venta en Bilbao" del director general de Bilbao Next en 2010, programa municipal estratégico ideado que asesorados por sociólogos y arquitectos, construían discursos en los que se empleaban grandes significantes, como "arte", "cultura", "lo simbólico", la "identidad" o la "memoria", para la legitimación de estrategias de "regeneración" y "revitalización" de espacios urbanos. Ya en 2010 se anunciaba desde tales instancias el paso a la "tercera revolución", la del conocimiento, que en sus discursos abandona el acento sobre la arquitectura, el urbanismo y el arte, para desplazarlo hacia la innovación, la tecnología y el diseño los cuales, ampliados al territorio vasco, pretenderían suponer una diferencia ante el modelo productivo de China.

Como acaba de indicarse, resulta peculiar el hecho de que la llamada sociedad del conocimiento deje en segundo plano al arte y la arquitectura, ya que los planteamientos modernos apropiados en forma de discurso por instancias estratégicas se referían precisamente a estas disciplinas capaces de articularse transformadoramente con la comunidad. Sin embargo, el propio hecho de la apropiación de estos discursos modernos para el cumplimiento de indicadores en las nuevas economías nos obliga a preguntarnos sobre el verdadero papel que han jugado recientemente el arte y la arquitectura en los procesos de transformación no sólo urbana, sino también cultural y económica.

En este sentido, consideramos especialmente oportuno que, en la denominada sociedad del conocimiento, el lugar que ocupan el arte y la arquitectura en la sociedad pueda evaluarse desde una perspectiva gnoseológica que tome en consideración la especificidad de estos saberes, no sólo en su particularidad disciplinar, sino también en la posición que ocupan respecto de los demás saberes conformadores de Cultura, entendida ésta como conjunto de sistemas de creencias, normas y valores naturalizados que mantienen cohesionado el orden de representación de una sociedad. 


\section{LA LECCIÓN DE LA MODERNIDAD: SÍNTESIS DE LAS ARTES}

Esta perspectiva no es en absoluto nueva, de hecho formaba parte indisociable del origen de las vanguardias y, a partir de éstas, de una línea caracterizada por el deseo de integrar distintos saberes disciplinares en la construcción del espacio común vivido: lo que unía al constructivismo con el neoplasticismo, la Bauhaus y los Congresos Internacionales de Arquitectura/CIAM, fue la convicción de que el arte, en connivencia con la arquitectura, era capaz de recuperar su relación orgánica con la sociedad siendo, además, agente de su transformación, ocupando una posición equitativa a los demás saberes al mismo tiempo que preservaba su especificidad.

Uno de los casos más paradigmáticos y cercanos lo supone el escultor Jorge Oteiza (1908-2003), artista cuya trayectoria entronca con esta larga tradición en la que el ensayo de la integración arte-arquitectura deviene en proyecto político de transformación social y cultural. No obstante, desde la década de 1960, y mediante el concepto de "Estéticas Aplicadas", Oteiza se diferenciará de los planteamientos de otros artistas que colaboran con la arquitectura y el urbanismo al defender que no deben añadirse más obras de arte a la ciudad, ya de por sí altamente expresiva y cargada de estímulos estéticos que conducen la vida del ciudadano y responden por él. Por el contrario, el modo óptimo de integración arte-arquitectura planteada por el escultor, y que deviene de su Propósito Experimental en escultura marcadamente espacialista, supondría la aplicación directa del saber del arte en la misma construcción espacial; se trata por tanto de la neutralización del alto grado de expresión estética urbana mediante el levantamiento de espacios "receptivos" en los que el ciudadano tenga un margen de maniobra estético suficiente para restituir su conciencia civil. Ante el reconocimiento de que la práctica del arte en los museos y galerías es incapaz de transformar la sociedad, Oteiza manifiesta abandonar la práctica escultórica en favor de proyectos de colaboración con arquitectos cuyo ideario será concretado por primera vez en 1958 en la conferencia Ciudad como obra de arte o en la Memoria del Monumento a José Battle y Ordóñez un año más tarde.

Este modo de integración entre arte y arquitectura tiene como referencia a las vanguardias rusas, y suponen al mismo tiempo la traslación del saber devenido de la disciplina escultórica hacia el nuevo ámbito de actuación que suponía la ciudad, desde una perspectiva gnoseológica que plantea el lugar del arte en la construcción social y cultural. Desde la especificidad propia del arte, 
Oteiza continúa suponiendo hoy una herramienta metodológica que, lejos de un acercamiento devoto y traducido debidamente a nuestro tiempo, aún incide en aspectos clave sobre los que estamos tratando. Gran parte de este ideario de integración arte-arquitectura descansa igualmente en la definición de su "Estética objetiva", en la que determinaba los factores y operaciones fundamentales para la creación del "Ser estético" -lo común a toda obra de arte. Aún mediante el lenguaje de su tiempo, advertimos la proximidad de sus planteamientos al advertirnos sobre el hecho de que la "razón vital" del artista -su expresión individual-, si bien suponía un factor más implicado en la operación de la creación, debía quedar técnicamente despejada para que emergiera la "razón existencial" -que atañe al otro. Es decir, contra el modo de hacer en arte como expresión de un yo individual, Oteiza es consciente de que el deseo del artista y la particularidad de su cultura son factores fundamentales en la operación, si bien para que ésta pueda dar con aquel espacio común de la comunidad, y por tanto político, el yo expresivo del artista debe reducirse en la operación creadora para que el espacio generado sea, en sus propia palabras, espacialmente "receptivo", para garantizar una identificación estética que remite a cierto espacio común el cual, avanzamos, se encontraría más acá de determinaciones ideológicas culturales.

\section{AMBIVALENCIA ESTÉTICA EN EL ESPACIO COMÚN}

Al igual que en los planteamientos formalistas rusos del arte, ese espacio "receptivo" supondría una discontinuidad de la realidad percibida, un modo de ver, pensar, y sentir distinto. Como indicábamos, esta perspectiva redunda en un planteamiento de la creación que va más allá de las particularidades disciplinares, orientado desde unos presupuestos gnoseológicos que pretenden distinguir su especificidad en relación con los modos naturalizados de percibir el mundo.

En este sentido, la relación arte-arquitectura señala aquel espacio común, público, que según vamos viendo, guardaría desde la perspectiva aquí planteada su propia particularidad. La misma noción de espacio público, o espacio común desde una perspectiva gnoseológica de la creación, nos llevaría a abordarlo no sólo en lo relativo al entorno material de la ciudad y sus espacios comunes, sino a reconocerlo como un espacio compartido de representación 
imaginaria (Castoriadis) desde la cual se instituyen aquellos modos naturalizados de percibir y actuar en el mundo, es decir, de la cultura, no exenta de tensiones y pugnas.

Las culturas son estructuras dictatoriales de transmisión de información que marcan las formas de sentir y de pensar el mundo. Unas claves de interpretación a cambio del empleo de sus signos, que dejan de lado un ámbito, real, que no pertenece a la cultura, un resto excultural o incultural, que queda fuera.

(Moraza en Rementeria, 2017: 536).

El empleo naturalizado de los signos se caracterizaría como continuidad en el flujo de las culturas, entendidas como espacios de convergencia y divergencia entre distintas identidades. Sin embargo, seguimos avanzando al indicar que, desde la perspectiva planteada, los fenómenos estéticos pueden guardar en esta dimensión pública y política cierto carácter de ambivalencia; si bien los fenómenos estéticos contribuyen a la continuidad del flujo naturalizado de los signos que constituyen una cultura, también hay fenómenos estéticos que, como discontinuidades, suponen una desnaturalización de los modos de percepción.

En la tradición moderna tendría que ver con el momento en el que hay un reconocimiento por parte de una cultura en la que hay una conciencia de que ese resto debería tener una función dinámica dentro de la cultura. Como si ese resto fuese una "singularidad" [...] en el sentido de resistencia a la cultura, aún sabiendo que perteneces a ella y que ella te pertenece a ti. Ese punto de resistencia como punto de emergencia de lo más real de un sujeto en lo que tiene de excultural e incultural es a lo que se puede referir Oteiza, y otras tantas personas. [...] Hoy en día, la finalidad de cualquier sistema coercitivo cultural es eliminar, domesticar ese resto $y$, en este punto, las cosas han avanzado mucho.

(Moraza en Rementeria, 2017: 537).

Por tanto, tal discontinuidad estética remitiría a aquel ese resto que precisamente podríamos caracterizar como espacio común por cuanto se encontraría más acá de toda construcción ideológico-cultural. Es en este sentido, como el espacio común, público, se dirime en la relación de lo estético con lo político, si bien aquí habría que distinguirlo del arte llamado político, que se caracterizaría por evocar representaciones de contenido propiamente político, es decir, vinculado a una ideología concreta. Tanto en el arte político como en el arte 
sin necesidad de epítetos, lo político de la creación pasará por dar, gracias a la operación técnica creadora, con ese espacio común que a todos nos implica.

Podría parecer paradójico afirmar que lo común se da en cuanto discontinuidad, pero habríamos de fijarnos en que la continuidad se da precisamente en el flujo de la particularidad y la diferencia, en los distintos modos y maneras de pensar, sentir, y actuar la vida, en las ideologías determinadas. En este sentido, desde el ámbito del saber creador, y aludiendo a Castoriadis, podríamos identificar una función "instituida" en cuanto redunda en la continuidad de la percepción naturalizada de la Cultura, contribuyendo a los modos instituidos de representación; y una función "instituyente", por cuanto abre el espacio común de ese real compartido más allá de determinaciones culturales, poniendo estéticamente en cuestionamiento los modos de representación culturales. La efectividad instituyente de las representaciones artísticas no consistiría, por tanto, en la transmisión exitosa de una determinada ideología cultural, o un contenido específico, sino en la constatación de que el espacio común y público se da como falla de toda representación, en su condición precaria, contraria al naturalismo pretendido por la Cultura.

Podríamos reconocer también cómo esta ambivalencia puede apreciarse igualmente en las relaciones entre arte y la arquitectura al margen de su categoría disciplinar (artista visual, arquitecto...), es decir, en tanto creadores. Aunque, más allá de oposiciones binarias, distinguimos que, en su carácter de ambivalencia, ambas funciones podrían darse al unísono; es decir, reconocer el acto radical que supone una discontinuidad, al mismo tiempo que la Cultura es capaz de otorgarle un sentido determinado e incluso contenido específico para poder reintegrarla de nuevo a la cadena de las significaciones culturales. Esto nos llevaría prácticamente a la idea de la creación (en arte, en arquitectura) como contra-monumento, entre una función cultural instituida -que da cuenta de su época e ideología, y una función artística instituyente -que trasciende su tiempo y lugar.

\section{TRANSMISIÓN Y EDUCACIÓN ESTÉTICA}

No obstante, la relación entre estética, creación e ideología conlleva una alta complejidad en la que aquí tampoco nos concierne entrar. Baste decir que la perspectiva de la creación que hemos convenido en definir como gnoseoló- 
gica ha sido asumida de forma efectiva en proyectos de integración arte-arquitectura desde la modernidad hasta nuestros días y que, de nuevo, tenemos como ejemplo particular no construido el Instituto de Investigaciones Estéticas que el propio Oteiza preveía poner en funcionamiento en los proyectos de colaboración con arquitectos. El Instituto pretendía, desde una formulación propia de su tiempo, contemplar la creación no sólo en sus particularidades disciplinares, sino como saber específico en relación con los demás saberes que conforman una cultura.

En este sentido, y al igual que en los albores de la modernidad arquitectónica (pensemos en Bruno Taut y La corona de la ciudad) el destino de la alianza arte-arquitectura partía del planteamiento de un modelo experimental de ciudad donde quedaba implícita la reorganización de los saberes. En Oteiza, el Instituto de Investigaciones Estéticas pretendía garantizar una transmisión de la especificidad del saber creador, que no debiera estar únicamente en manos de los artistas (al margen de su disciplina: escultura, pintura, arquitectura...), sino de toda la comunidad, a través de una "educación estética" como único garante de una sociedad verdaderamente emancipada.

La última oportunidad de llevar a cabo este proyecto de integración arte-arquitectura en tanto modelo experimental de ciudad en el que estaba implicada la idea de una transmisión del saber creador nos lleva de nuevo a la ciudad de Bilbao, concretamente al proyecto malogrado de Centro Cultural para la Alhóndiga de Bilbao en 1988, momento previo a las transformaciones indicadas al inicio de este artículo. Fue la primera iniciativa institucional para la reconversión económica y cultural de la ciudad, que contó con Oteiza y los arquitectos Juan Daniel Fullaondo y Fco. Javier Sáenz de Oiza. Este proyecto, sin ser realizado, representa un gozne, un momento de discontinuidad entre dos modos instituidos de entender y actuar en el mundo; un lugar en el tiempo que, en su condición de bisagra, contenía en sí mismo el germen de toda posibilidad, pero que condensaba igualmente las diferencias encontradas entre los últimos coletazos de una tardomodernidad representada por Oteiza, Fullaondo y Sáenz de Oiza, y los artistas jóvenes que pretendían una reformulación del proyecto moderno acorde al nuevo paradigma posmoderno. Una reformulación que, de hecho, no olvidaría sino que incidiría igualmente en esta perspectiva gnoseológica del arte, la cual podemos comprobar incluso en artistas de generaciones posteriores que organizan, acorde a las especificidades de su tiempo, espacios para la transmisión de la especificidad del saber creador. La principal diferencia estará en el alcance: mientras que las pretensiones moderna y tardomoderna pretendían alcanzar a la ciudad y su 
comunidad, desde la posmodernidad en adelante la transmisión de ese saber se reducirá exclusivamente a la comunidad artística.

\section{REDEFINICIONES}

Continuar la perspectiva gnoseológica de la relación arte-arquitectura hoy en día implica necesariamente contemplar la ambivalencia estética y la necesidad de redefinición epocal, aspectos ambos que redundarían tanto en el reconocimiento del espacio común de la creación como, a partir de aquí, en la reformulación de las particularidades disciplinares de acuerdo a las características propias de nuestra época cargada de incertidumbre. La cultura, nuestra cultura, así, será el material (siempre lo ha sido) ante el cual articularnos en relación a sus particularidades, requerimientos y resistencias. $Y$ los creadores (artistas, arquitectos...) responderán de forma particular desde una ideología en arte - la cual determinará los procesos a seguir en la operación creadora, como una ideología para el arte, caracterizada por la lucha cultural para que lo propio de la creación pueda darse, todo lo cual determinará la especificidad de cada creador que, en su búsqueda del espacio común, bordeará los límites de lo pensable.

\section{Referencias}

Arnaiz, Ana, Jabier Elorriaga, Xabier Laka \& Jabier Moreno. (2008). La Colina Vacía. Jorge Oteiza-Roberto Puig. Monumento a José Batlle y Ordóñez: 1956-1964. Bilbao: ehupress/ FMJO.

Arnaiz, Ana \& Iskandar Rementería. (2014). "Saber del escultor entre El Arte y la Ciudad". Art \& Sensorium, Revista Interdisciplinar Internacional de Artes Visuais, Vol. 1, N. 1, Junho 2014. Curitiba (PR-Brasil): EMBAP/Universidade Estadual do Paraná. http://periodicos.unespar. edu.br/index.php/sensorium/article/view/200

Castoriadis, Cornelius. (1983). La institución imaginaria de la sociedad. vol I y II. Marxismo y teoría revolucionaria, Barcelona: Tusquets.

Laka, Xabier (2010) Síntesis de las artes. Relaciones Escultura/Arquitectura. Experiencia AzterIan. Departamento de Escultura. Universidad del País Vasco/EHU, (Tesis doctoral)

Garcés, Marina. (2017). Nueva ilustración radical. Barcelona: Anagrama.

Oteiza, Jorge. (1965). "El arte como escuela política de tomas de conciencia" en Oteiza, Jorge (1984) Ejercicios espirituales en un túnel. De Antropología estética vasca y sobre nuestra recuperación política como Estética aplicada. Zarautz: Hórdago, 1984; pp 44-145. 
Taut, Bruno. (1919) 1997. "La corona de la ciudad", en Bruno Taut. Escritos expresionistas. Madrid: El Croquis (Iñaki Ábalos ed.)

Rementeria, Iskandar. (2017). Oteiza y el Centro Cultural Alhóndiga de Bilbao (1988). Una interpretación estética. Pamplona: Fundación Museo Jorge Oteiza.

\section{Notas}

${ }^{1}$ En la sesión del 13 de diciembre de 2010, el Consejo Cívico del Ayuntamiento de Bilbao se reunió junto al sociólogo, arquitecto y presidente de la Fundación Metrópoli, Alfonso Vegara, y al ex consejero de Economía del Gobierno vasco, Pedro Luís Uriarte. Los dos principales objetivos de esta sesión fueron, por un lado, concretar las labores del entonces nuevo organismo Bilbao Next, y por otro, permitir que los invitados-asesores presentaran sus conferencias, las cuales versaron sobre la situación económica global, pretendiendo así motivar la reflexión sobre las posibles estrategias para el futuro de Bilbao. 